\title{
ELETRENCEFALOGRAMA NOS TRAUMATISMOS CRANIO-ENCEFALICOS
}

\author{
LINEU CORRÊA FonsECA *
}

O avanço tecnológico do mundo moderno está sendo acompanhado pela crescente exposição do homem a diferentes tipos de traumatismos e o trauma crânio-encefálico passou assim, nos grandes centros, a ser uma das principais causas de mortalidade e morbidade, advindo daí a importância do estudo de todos os meios diagnósticos úteis na sua avaliação.

Williams (1941) ${ }^{26,27}$ e autores subseqüentes $6,7,8,11,13,15,16,20,21,22,23$, 24, 25 descreveram alterações eletrencefalográficas nos traumas crânio-encefálicos recentes e nos estados crônicos pós-traumáticos, encontrando nítida correlação com a severidade da lesão. Assinalaram que um eletrencefalograma (EEG) anormal parece refletir o grau de disfunção cerebral, sugerindo uma anormalidade orgânica, constituindo, por isso, um método auxiliar de exploração.

Nosso trabalho tem por objetivo rever a importância do EEG na avaliação de pacientes com traumatismo crânio-encefálico.

\section{CASUISTICA E METODOS}

Estudamos 387 pacientes com traumatismo crânio-encefálico internados em dois centros hospitalares de Campinas ou atendidos em nivel ambulatorial. A idade esteve compreendida entre 9 meses e 85 anos, sendo 289 do sexo masculino e 98 do sexo feminino. Para cada faixa etária houve nitido predominio do sexo masculino com exclusão da faixa etária de 0 a 10 anos em que não houve diferença estatisticamente significante na proporção de individuos de ambos os sexos (Quadro 1). Em 337 pacientes houve traumatismo crânio-encefálico fechado, aberto em 10 e 40 foram operados de hematoma intracraniano. Vários pacientes foram submetidos a mais de um EEG, obtendo-se no total 551 exames. O tempo entre o trauma e a realização do EEG encontra-se representado no quadro 4.

Os EEGs foram, sempre que possivel, realizados durante vigília, com ativação pela hiperpnéia durante 3 minutos, e muitas vezes durante sono espontâneo ou induzido por barbitúrico (Secobarbital $10 \mathrm{mg} / \mathrm{kg}$ de peso corporal). O aparelho utilizado foi um Kaiser, modelo 55, de 8 canais, com disposição de eletrodos aconselhada pela Federação Internacional para Eletrencefalografia e Neurofisiologia Clínica (método 10-20). A velocidade de registro do papel inscritor foi de $3 \mathrm{~cm} / \mathrm{seg}$. e a constante de tempo entre 0,2 e 0,5 .

* Neurologista e eletrencefalografista do Centro Médico de Campinas (SP).

Nota do autor - Agradeço a colaboração do Dr. Adail Freitas Julião pelo incentivo e orientação, do Dr. Walter Pinto Júnior pela orientação e revisão estatística e do Dr. Roque José Balbo, que possibilitou a avaliação eletrencefalográfica em estreita correlação com os dados clínicos, da maior parte dos pacientes desta série. 
Os seguintes dados clínicos foram relacionados com os achados eletrencefalográficos: idade, tempo após o trauma, duração da perda de consciência, presença de sinais focais ao exame neurológico, aparecimento de convulsões, existência de trauma crânio-encefálico aberto ou hematoma, líquido cefalorraqueano hemorrágico e fratura de crânio.

\section{RESULTADOS}

Dos 387 pacientes, $237(61,24 \%)$ tiveram pelo menos um EEG alterado e 150 $(38,75 \%)$ apresentaram sempre exames normais. Dos 551 exames, $353(64,06 \%)$ foram normais.

Tipos de anormalidade - Sob a denominação de "anormalidades lentas continuas difusas" (ALCD) foram incluidas as anormalidades contínuas ou quase contínuas, difusas, com ou sem predomínio focal, compreendendo desde a organizaçāo discreta com pequeno contingente de ondas teta, até o sofrimento difuso acentuado caracterizado por ondas delta (Figs. $1,2,3$ e 4). Quando essas anormalidades eram focais ou de predominio focal (Figs. $5,6 \mathrm{e} 7$ ), foram referidas como "anormalidades lentas continuas focais" (ALCF). Quando as anormalidades eram difusas mas havia algum tipo de predominio focal eram referidas simultanemente como "anormalidades lentas continuas difusas" e como "anormalidades lentas continuas focais".

As ALCD apareceram em 173 (31,45\%) EEGs, constituindo-se na anormalidade mais freqüente. Em 102 EEGs encontrou-se associação com ALCF ou outras alterações, sendo isolada no restante.

As ALCF, associadas ou não a outras alteraçōes, foram registradas em 149 $(27,04 \%)$ EEGs, sendo que, em 66 todo um hemisfério participava do sofrimento, não permitindo melhor localização. Em 31 EEGs, o sofrimento foi parietoccipital, em 45 temporal e em 7 frontocentral.

As depressões (Fig. 8) focais ou difusas foram encontradas em 29 (5,25\%) EEGs.

Em 110 (19,96\%) EEGs constatou-se anormalidade paroxística (Figs, 9 e 10), sendo por ondas lentas em 70 e por ondas agudas ou pontas em 40. Predominaram nas regiões temporais (70 EEGs), mas ocorreram de modo difuso em 22, na região parietoccipital em 9 e frontocentral em 9.

Idade - A proporção de exames anormais foi semelhante nas várias faixas etárias, excluindo a de 0 a 5 anos, onde ela foi menor (Quadro 2).

No quadro 3 estão assinalados os vários tipos de anormalidades lentas contínuas, nas diferentes faixas etárias, tendo-se eliminado eventuais exames similares de um mesmo paciente.

A anormalidade lenta continua parietoccipital (Fig. 7) foi mais comumente encontrada abaixo dos 10 anos, o mesmo ocorrendo com a anormalidade lenta continua frontocentro-temporal em pacientes com idade superior a 10 anos. Tal achado pode ser comprovado pela alta significância estatistica encontrada $\left(\chi^{2}=23,414\right.$; 1 G.L.; $P<0,001)$. Há também diferença significativa ao comparar as mesmas anormalidades nas faixas de até 10 anos, com a de 11 a 20 anos e com a de mais đe 20 anos $\left(\chi^{2}\right.$ com correção de Yates $\left.=20,249 ; P<0,001\right)$, demonstrando, assim, a progressiva diminuição das anormalidades parietoccipitais com o aumentar da idade (Quadro 3).

Nas demais alterações ao EEG não evidenciamos diferenças estatisticamente significativas.

Tempo entre EEG e o traumatismo - Quanto maior o intervalo de tempo entre o trauma e o EEG menor é a proporção das anormalidades no 1.0 ano, havendo, depois, pequena elevação. Embora quando comparamos os grupos próximos não tenhamos constatado diferença estatisticamente significante, 0 mesmo não podemos 

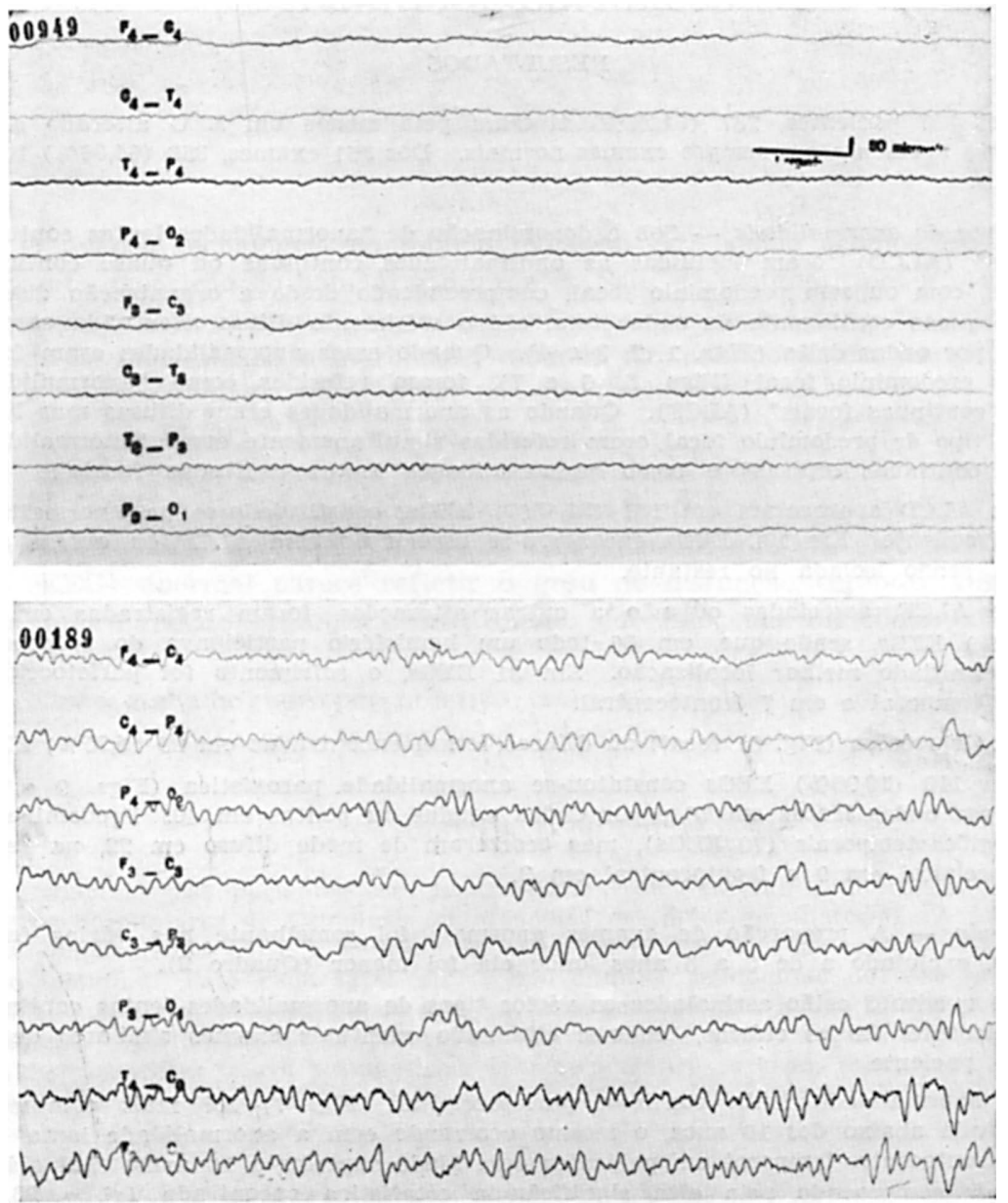

Fig. 1 - Caso 101. Sexo masculino, 22 anos de idade. Ritmo dominante 7 $c / s$ com pequeno contingente associado de ritmos rápidos em areas anteriores.

Fig. 2-Caso 188. Sexo masculino, 28 anos. Constituido por ritmos 6-7 $\mathrm{c} / \mathrm{s}$ com certo contingente mais lento intercalado. 


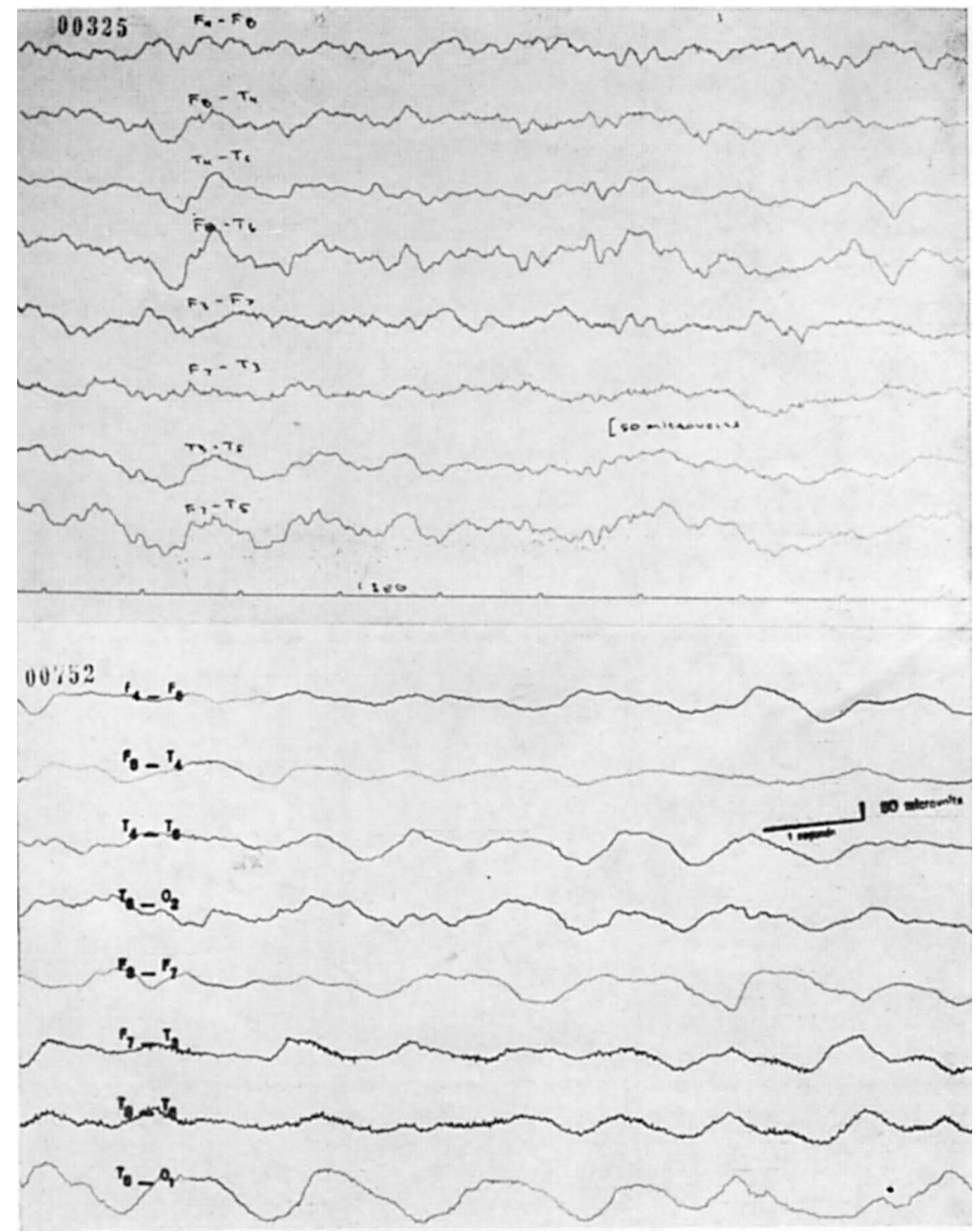

Fig. 3 - Caso 54. Sexo feminino, 13 anos. Constitutdo por ondas delta e teta associadas de modo irregular.

Fig. 4 - Caso 126. Sexo masculino, 25 anos. Constituido por ondas delta difusas. Não há reação a estímulos. 

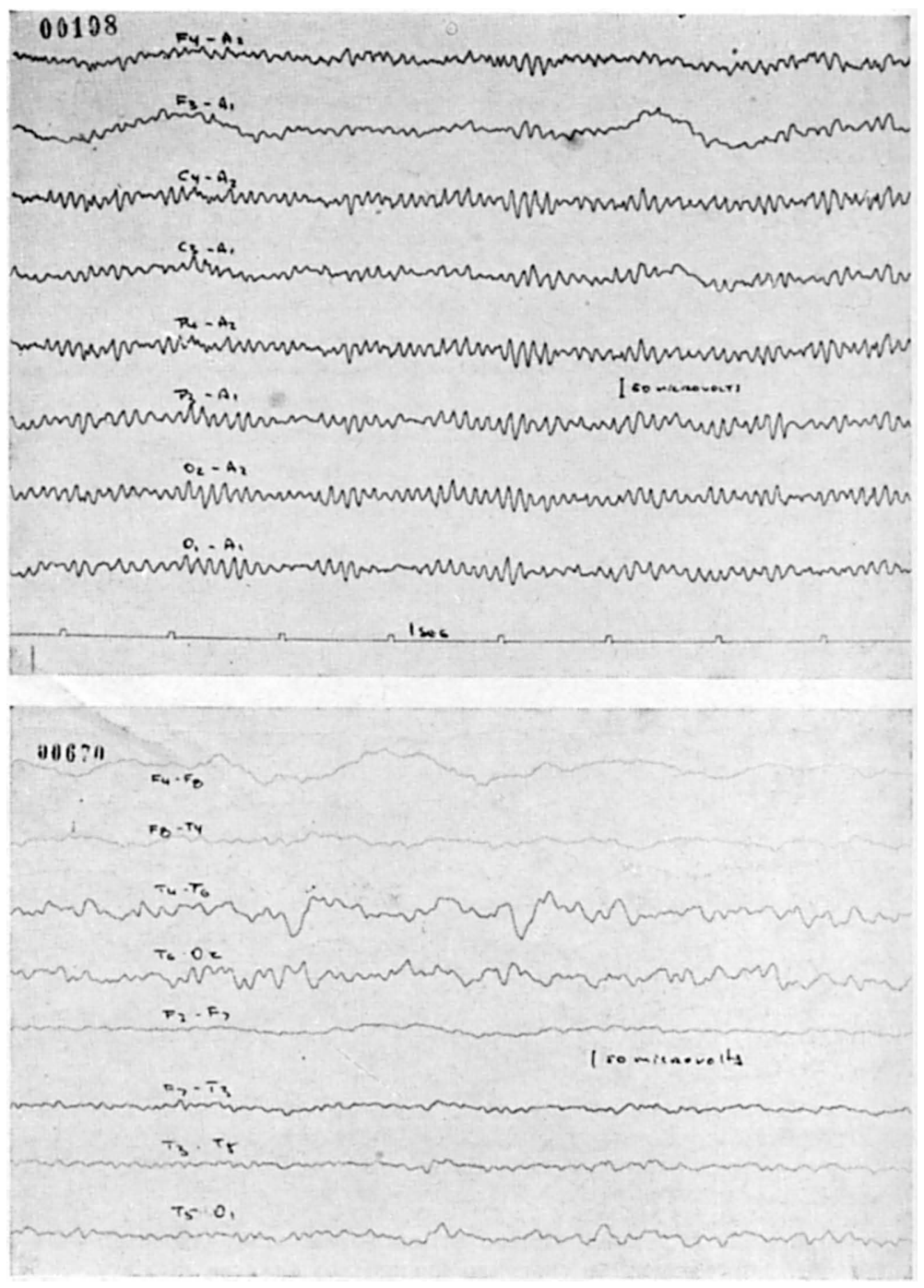

Fig. 5 - Caso 56. Sexo masculino, 35 anos. Ritmo dominante $10 \mathrm{c} / \mathrm{s}$. Na região central e particularmente frontal esquerda intercalam-se ondas delta de médio potencial.

Fig. 6 - Caso 364. Sexo masculino, 14 anos de idade. Anormalidade contínua difusa, mas com franco predomínio pelo hemisfério direito, caracterizada pelo maior contingente de ondas lentas. 

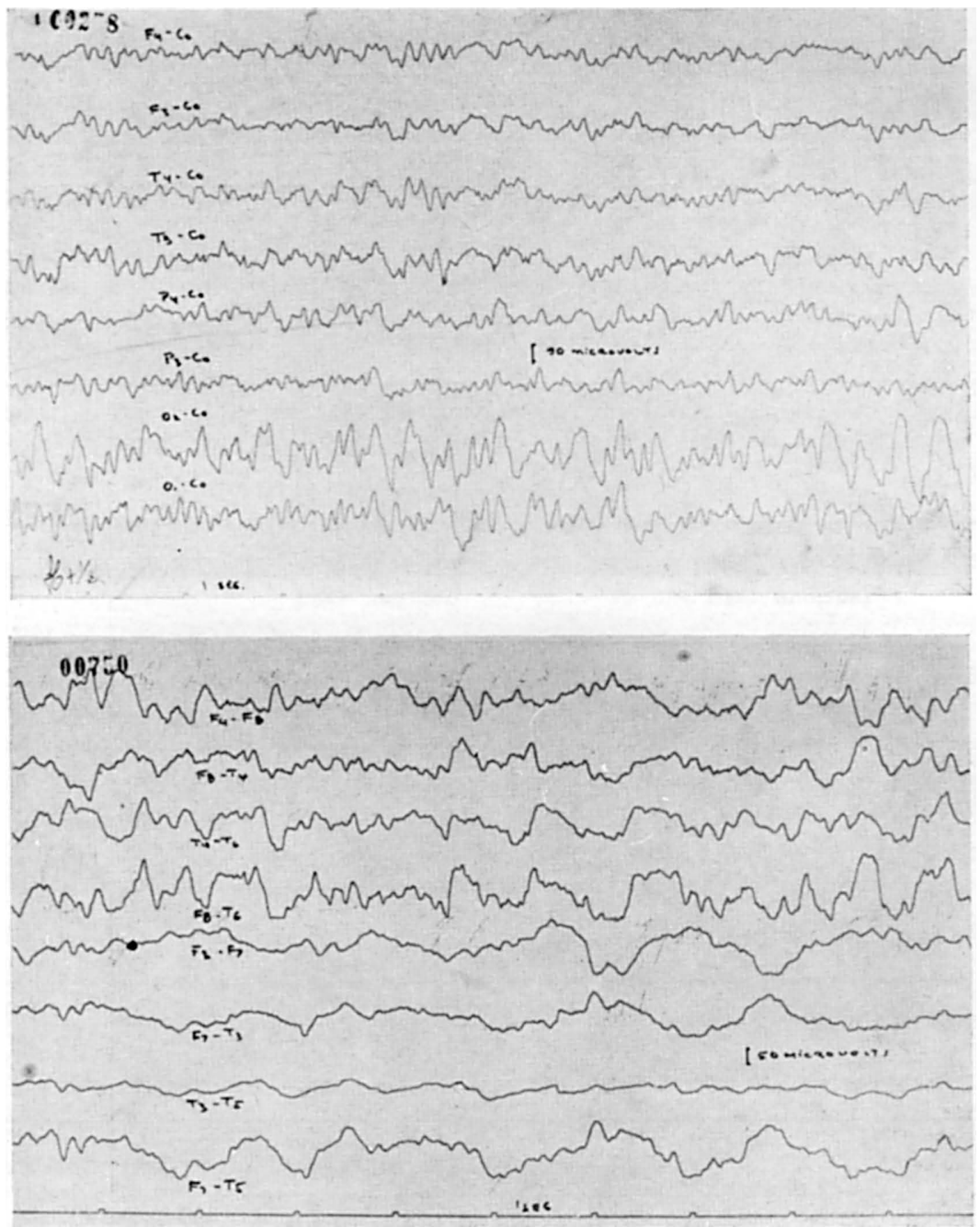

Fig. 7 - Caso 2s5. Sexo masculino, 10 anos de idade. Desorganizacão da atividade elétrica cerebral na região occipital direita, determinada por ondas lentas.

Fig. 8-Caso $\$ 80$. Sexo masculino, 69 anos. No hemisfério direito dominam ondas $6-7 \mathrm{c} / \mathrm{s}$ e no esquerdo se evidenciam principalmente ondas mais lentas, porém com amplitude pouco elevada. Trata-se de paciente com hematoma subdural crônico esquerdo. 

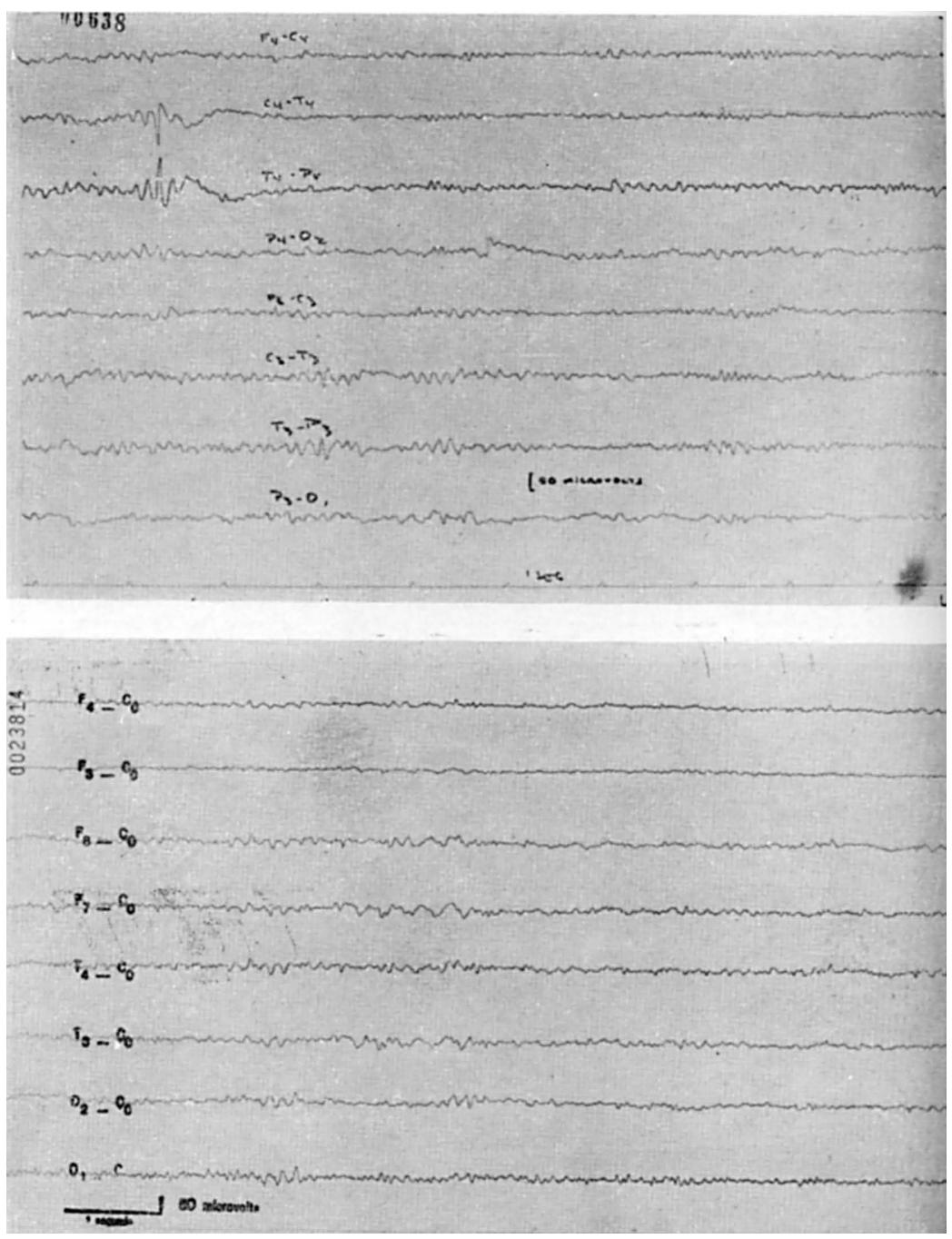

Fig. 9 - Caso 364. Sexo masculino, 14 anos de idade. Anormalidade paroxística nas regiões temporais do hemisfério direito constituida por ondas de aspecto agudo.

Fig. 10 - Caso 52. Sexo masculino, 44 anos de idade. Anormalidade paroxistica nas regióes temporais do hemisfério esquerdo por surtos de ondas $4 \mathrm{c} / \mathrm{s}$. 


\begin{tabular}{|c|c|c|c|c|c|c|}
\hline \multirow[t]{3}{*}{ Idade em anos } & \multicolumn{4}{|c|}{ Pacientes } & \multicolumn{2}{|c|}{ Totais } \\
\hline & \multicolumn{2}{|c|}{ masculinos } & \multicolumn{2}{|c|}{ femininos } & \multirow[b]{2}{*}{ N.o } & \multirow[b]{2}{*}{$\%$} \\
\hline & N.o & $\%$ & N.o & $\%$ & & \\
\hline Até 10 & 49 & 58,33 & 35 & 41,66 & 84 & 21,71 \\
\hline $11-20$ & 57 & 77,03 & 17 & 22,97 & 74 & 19,12 \\
\hline $21-30$ & 64 & 77,11 & 19 & 22,89 & 83 & 21,45 \\
\hline $31-40$ & 50 & 79,37 & 13 & 20,63 & 63 & 16,28 \\
\hline $41-50$ & 24 & 88,89 & 3 & 11,11 & 27 & 6,98 \\
\hline $51-60$ & 19 & 86,36 & 3 & 13,64 & 22 & 5,68 \\
\hline mais de 61 & 26 & 76,47 & 8 & 23,53 & 34 & 8,79 \\
\hline Totais & 289 & 74,68 & 98 & 25,32 & 387 & 100,01 \\
\hline
\end{tabular}

Quadro 1 - Distribuição dos 387 pacientes com traumatismo crânio-encefálico segundo a faixa etária e o sexo.

\begin{tabular}{|c|c|c|c|c|c|c|c|c|}
\hline \multirow[t]{4}{*}{ Idade em anos } & \multicolumn{3}{|c|}{$\begin{array}{l}\text { Pacientes com } \\
\text { EEG normal }\end{array}$} & \multicolumn{5}{|c|}{$\begin{array}{c}\text { Pacientes com pelo menos } \\
\text { um EEG anormal }\end{array}$} \\
\hline & \multirow[t]{3}{*}{ N.o } & \multirow{2}{*}{\multicolumn{2}{|c|}{$\begin{array}{l}\text { EEGs } \\
\text { realizados }\end{array}$}} & \multirow[t]{3}{*}{ N.o } & \multicolumn{4}{|c|}{ EEGs } \\
\hline & & & & & \multicolumn{2}{|c|}{ normais } & \multicolumn{2}{|c|}{ anormais } \\
\hline & & N.o & $\%$ & & N.o & $\%$ & N.o & $\%$ \\
\hline Até $\mathbf{5}$ & 25 & 29 & 46,03 & 19 & 4 & 6,35 & 30 & 47,72 \\
\hline $6-10$ & 12 & 12 & 22,22 & 28 & 4 & 7,41 & 38 & 70,37 \\
\hline $11-20$ & 23 & 23 & 20,91 & 51 & 8 & 7,27 & 79 & 71,82 \\
\hline mais de 20 & 90 & 98 & 30,25 & 139 & 20 & 6,17 & 206 & 63,58 \\
\hline Totais & 150 & 162 & 29,40 & 237 & 38 & 6,53 & 353 & 64,07 \\
\hline
\end{tabular}

\begin{tabular}{|c|c|c|c|c|c|c|c|c|c|}
\hline \multirow[t]{2}{*}{$A L C$} & \multicolumn{8}{|c|}{ Idade em anos } & \multirow[t]{2}{*}{ Totais } \\
\hline & N. ${ }^{A}$ & te $\begin{array}{l}5 \\
\%\end{array}$ & $\begin{array}{r}6 \\
\text { N.o }\end{array}$ & $-\begin{array}{c}10 \\
\%\end{array}$ & $\begin{array}{l}11 \\
N . o\end{array}$ & $-\begin{array}{c}20 \\
\%\end{array}$ & $\begin{array}{l}\text { mais } \\
\text { N.o }\end{array}$ & $\begin{array}{c}\text { de } 20 \\
\%\end{array}$ & \\
\hline Difusa & 7 & 5,51 & 14 & 11,02 & 26 & 20,47 & 80 & 62,99 & 127 \\
\hline De um hemisfério & 5 & 7,04 & 4 & 5,63 & 6 & 8,45 & 56 & 78,87 & 71 \\
\hline Parietoccipital & 8 & 26,66 & 13 & 43,33 & $\mathbf{3}$ & 10,00 & 6 & 20,00 & 30 \\
\hline Frontocentrotemporal & 2 & 5,71 & 2 & 5,71 & 9 & 25,71 & 22 & 62,85 & 35 \\
\hline
\end{tabular}




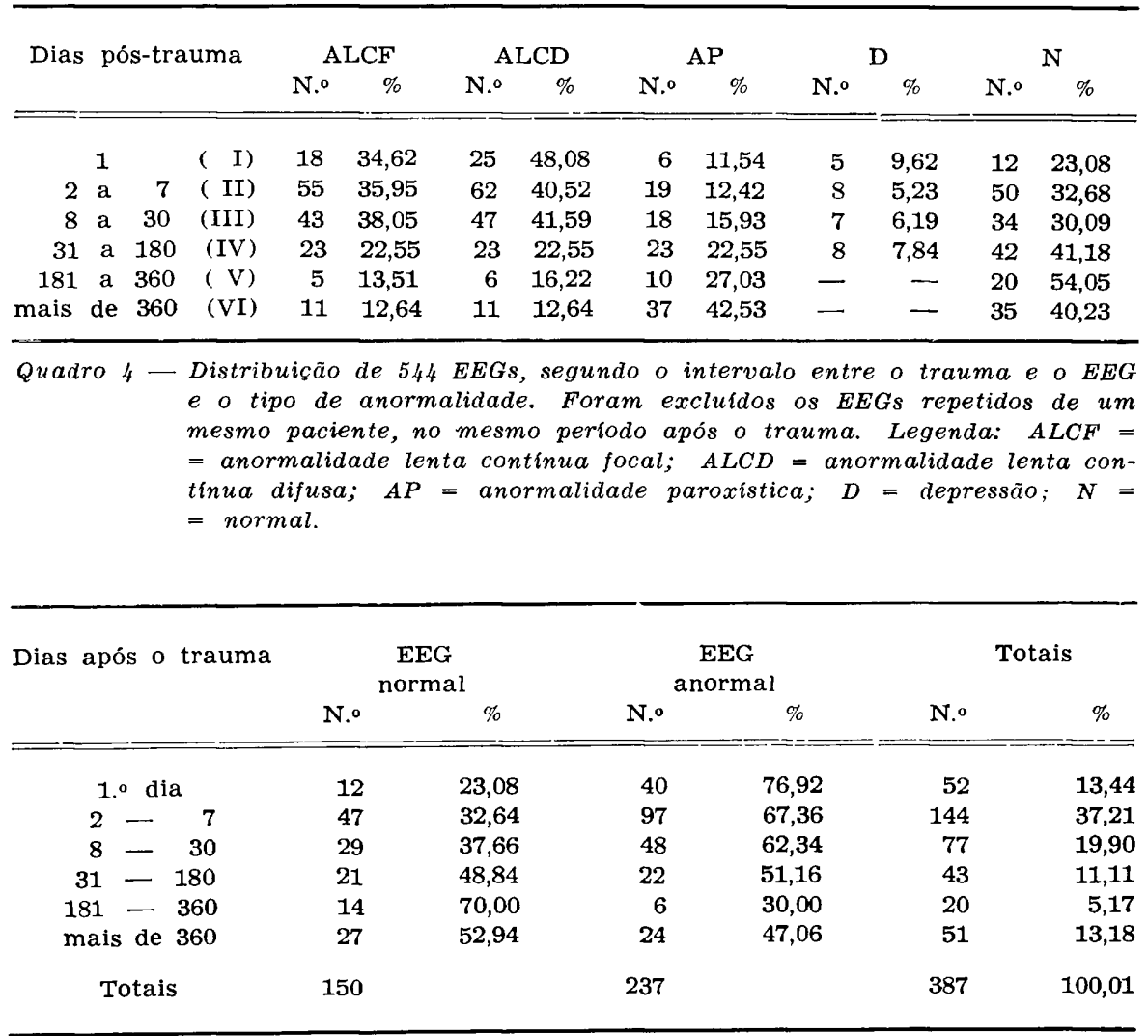

Quadro 5 - Distribuicão dos 387 pacientes segundo o intervalo de tempo entre o primeiro eletrencefalograma e o trauma e a presença de anormalidade.

Pacientes com EEG normal

Exames feitos

N. ${ }^{\circ} \quad$ N. $\circ \quad \%$
Pacientes com pelo menos um EEG anormal

Exames realizados

\begin{tabular}{ccc}
\hline \multicolumn{2}{c}{ normais } & \multicolumn{2}{c}{ anormais } \\
N. & $\%$ & N.o $\%$ \\
\hline
\end{tabular}

Sem perda de consciência (A)

Por menos de uma hora (B)

Mais de uma e menos de 24h (C)

Mais de 24 hs. (D)

Tempo indeterminado

(E)

Totais

$\begin{array}{rrrrrrrr}29 & 32 & 45,71 & 29 & 4 & 5,71 & 34 & 48,57 \\ 62 & 67 & 44,67 & 56 & 8 & 5,33 & 75 & 50,00 \\ 21 & 23 & 26,14 & 42 & 8 & 9,10 & 57 & 64,77 \\ 11 & 12 & 7,95 & 71 & 13 & 8,61 & 126 & 83,44 \\ 27 & 28 & 30,43 & 39 & 3 & 3,26 & 61 & 66,30 \\ 150 & 162 & 29,40 & 237 & 36 & 6,53 & 353 & 64,07\end{array}$

Quadro 6 - Distribuição dos 551 EEGs de 387 pacientes, segundo a perda de consciência e a presença de anormalidades. 


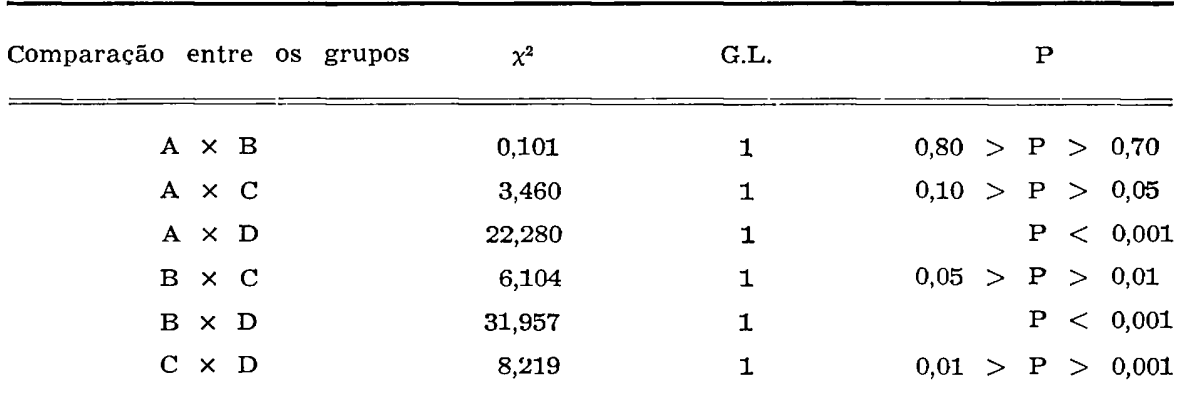

Quadro 7 -Comparação estatistica entre os diferentes grupos, segundo a perda de consciência $e$ o número de pacientes com EEG anormal (cf. Quadro 6).

\begin{tabular}{|c|c|c|c|c|c|c|c|c|}
\hline & \multicolumn{2}{|c|}{ HSA } & \multicolumn{2}{|c|}{$\mathrm{HI}$} & \multicolumn{2}{|c|}{$\mathrm{HE}$} & \multicolumn{2}{|c|}{ HSC } \\
\hline & Pré- & Pós- & Pré- & Pós- & Pré- & Pós- & Prẽ- & Pós- \\
\hline $\begin{array}{l}\text { Anormalidade lenta } \\
\text { continua difusa }\end{array}$ & 5 & 8 & 2 & 7 & 1 & 9 & 9 & 1 \\
\hline $\begin{array}{l}\text { Anormalidade lenta } \\
\text { continua focal }\end{array}$ & 3 & 6 & 1 & 11 & 3 & 8 & 8 & 5 \\
\hline $\begin{array}{l}\text { Anormalidade } \\
\text { paroxistica }\end{array}$ & 1 & 3 & - & 1 & 一 & 2 & 4 & 2 \\
\hline Depressão & 3 & - & - & - & - & - & 3 & 2 \\
\hline Normal & 2 & 2 & 一 & 4 & - & 2 & 1 & 4 \\
\hline Total de exames & 8 & 11 & 2 & 16 & 4 & 14 & 18 & 14 \\
\hline
\end{tabular}

Quadro 8 - Distribuição de 55 EEGs pré e pós-operatórios, segundo a anormalidade e o tipo de hematoma. Legenda: HSA = Hematoma subdural agudo; $H I=H e m a t o m a$ intracerebral; $H E=$ Hematoma extradural; HSC = = Hematoma subdural crônico; Pré- = pré-operatório; Pós- = pós-operatório.

\begin{tabular}{cccccc}
\hline Pacientes & EEG & normal & EEG anormal & Totais \\
& N.0 & $\%$ & N.o & $\%$ & 31 \\
\hline \hline Com hematoma & 2 & 6,45 & 29 & 93,54 & 347 \\
Sem hematoma & 146 & 42,07 & 201 & 57,92 & 378 \\
\hline
\end{tabular}

Quadro 9 - Distribuição dos 387 pacientes segundo a existância ou não de hematoma $e$ a presença de anormalidade. 


\begin{tabular}{lrccccc}
\hline \multirow{2}{*}{ Hematomas } & \multicolumn{3}{c}{ Lateralização } & Normal & Totais \\
\cline { 2 - 4 } & certa & impossivel & errada & & \\
\hline Subdural agudo & 6 & 1 & - & 1 & 8 \\
Intracerebral & 1 & 1 & - & - & 2 \\
Extradural & 3 & 1 & - & - & 4 \\
Subdural crônico & 10 & 2 & 3 & 1 & 16 \\
\multicolumn{1}{c}{ Totais } & 20 & 5 & 3 & 2 & 30 \\
\hline
\end{tabular}

Quadro 10 - Distribuição de 30 pacientes com hematoma unilateral, segundo o tipo de hematoma e possibilidade de lateralização ao EEG.

dizer ao compararmos os exames realizados no primeiro dia e os de 6 a 12 meses pós-trauma ( $\chi^{2}=9,009 ; 1$ G.L.; $\left.0,01>\mathrm{P}>0,001\right)$, o que demonstra a menor proporção de anormalidades neste grupo (Quadro 4).

Levando-se em conta apenas o primeiro EEG de cada paciente, também fica evidenciada a progressiva queda de proporção de EEGs anormais quanto mais distante do trauma é examinado o paciente, excluído o grupo após o $1 .^{\circ}$ ano (Quadro 5).

Há também modificações no tipo de anormalidade com o passar do tempo. Assim, as anormalidades lentas focais diminuem entre o $1^{\circ}$ e o $6 .^{\circ}$ mês, pois ao compararmos estatisticamente o grupo III e o grupo IV, eles diferem significativamente $\left(\chi^{2}=6,057 ; 1\right.$ G.L.; $\left.0,01>\mathrm{P}>0,001\right)$, decrescendo mais ainda essa proporção nos períodos ulteriores (Quadro 4). O mesmo ocorre com as anormalidades lentas continuas difusas, na comparação entre o grupo III e IV $\left(\chi^{2}=8,854 ; 1\right.$ G.L.; $0,01>\mathrm{P}>0,001$ ).

Podemos ver também, no quadro 4, que as anormalidades paroxisticas têm, pelo contrário, seu percentual progressivamente aumentado, com significância estatística, ao comparar o grupo III com o grupo IV $\left(\chi^{2}=17,444 ; 1\right.$ G.L.; $\left.\mathrm{P}<0,001\right)$ e o grupo IV com o grupo V $\left(x^{2}=8,650 ; 1\right.$ G.L.; $\left.0,01>\mathrm{P}>0,001\right)$.

As depressões ocorreram nos primeiros 6 meses após o trauma, não se prestando, porém, à análise estatística, dado o pequeno número de casos.

Procurando outros dados para a análise evolutiva das anormalidades eletrencefalográficas, separamos 28 pacientes com um EEG inicial anormal e um EEG final normal. Nestes, a normalização ocorreu em $4(14,29 \%)$ no $1.0^{\circ}$ mês, em $17(60,71 \%)$ no intervalo do $1^{\circ}$ ao $6^{\circ}$ mês, em $3(10,71 \%)$ entre o $6 .^{\circ}$ e o $12 .^{\circ}$ mês e em 4 $(14,29 \%)$ após o primeiro ano. As anormalidades iniciais eram, na maioria dos pacientes, continuas, constituidas por ondas lentas focais ou difusas.

Duração da perda de consciência - No quadro 6 separamos os pacientes em grupos segundo a presença ou não de perda de consciência e sua duração, relacionando-os aos achados eletrográficos.

A análise estatística entre os vários grupos do quadro 6 revela a significativa maior proporção das anormalidades (Quadro 7) nos pacientes que perderam a consciência por mais de 24 horas (D) em relação aos do grupo dos que a perderam entre uma hora e 24 horas (C) e destes em relação ao grupo dos que a perderam por menos de uma hora (B). Não houve diferença significativa entre o grupo sem perda de consciência e o com perda menor do que 24 horas. Pela falta de homogeneidade de seus componentes não foi avaliado estatisticamente o grupo com perda de consciência mal caracterizada (E). 
Em 42 pacientes foi obtido EEG durante coma, encontrando-se sofrimento difuso desde as ondas delta de baixa freqüência e monomórficas até os traçados de ondas teta, com certo contingente de maior freqüência intercalado. Em 25 pacientes as anormalidades tinham algum tipo de predominio focal e em 7 havia depressão focal. Em um paciente que mostrava, ao exame neurológico, hipertonia com hiperextensão dos quatro membros, sinal de Babinski bilateral, anisocoria e tendência ao desvio conjugado dos olhos para a esquerda, o EEG mostrou ritmos rápidos de baixa voltagem associados a certo contingente de ritmos 9-12 c/s, tornando dificil a distinção de um traçado normal. Contudo, a evolução dos EEGs mostrou sofrimento cerebral difuso por ondas delta e teta de elevada voltagem. Os "splindles", ondas agudas do vertex e complexos $\mathrm{K}$ apareceram em 4 pacientes. Um paciente em coma "depassé" apresentava traçado de silêncio elétrico.

Traumatismo crânio-encefálico aberto - Dos 10 pacientes com trauma crânio-encefálico aberto, 9 mostraram-se com EEGs anormais. O sofrimento focal foi constatado em 7 exames e as anormalidades paroxísticas em 4 dos 15 EEGs realizados. Cumpre realçar que 4 desses pacientes apresentaram crises convulsivas.

Aparecimento de convulsões - Entendendo por epilepsia pós-traumática aquela em que as crises convulsivas apareceram pela primeira vez após o trauma ${ }^{\circ}$, a mesma pôde ser observada em 43 pacientes, sendo em 18 na forma tardia (iniciada após a primeira semana) e 25 na forma precoce, das quais 7 imediatas.

As crises generalizadas apareceram em 16 pacientes, as motoras focais em 9 , as versivas em 4 e as do tipo temporal em 4 .

Em 8 desses pacientes havia hematoma intracraniano e em 4 trauma crânio-encefálico aberto.

Trinta e cinco doentes tiveram exames anormais e a anormalidade mais freqüente foi a paroxistica difusa ou focal, assinalada em 29 pacientes. Dos 72 EEGs realizados nesses pacientes, 15 foram normais, 38 tinham alteraçóes paroxísticas, 30 anormalidades lentas continuas difusas e 21 focais. Um dos exames mostrava atividade continua espicula-onda $2-4 \mathrm{c} / \mathrm{s}$ num hemisfério, com predominio frontotemporal, sem manifestação clínica concomitante, embora o paciente houvesse apresentado crise focal horas antes.

Existência de sinais focais ao exame neurológico - Em 96 pacientes havia, ao exame neurológico, a presença de sinais focais (deficit motor ou sensitivo, alterações dos nervos cranianos, afasia, hemianopsia) persistentes ou não durante a evolução. Des:e total, $80(83,33 \%)$ tinham alterações ao EEG e, por vezes, com exame neurológico já normalizado. Dos 171 EEGs realizados, 137 (80,11\%) foram anormais, constituidos na maioria por anormalidades lentas contínuas focais ou difusas.

Líquido cefalorraqueano hemorrágico - Em 35 pacientes com líquido cefalorraqueano hemorrágico, $31(88,57 \%)$ apresentaram EEGs anormais, constatando-se ALCF em 16 e ALCD em 25 dos 53 EEGs realizados.

Fratura simples de crânio - Dentre os 82 pacientes com fratura simples de crânio, $56(68,29 \%)$ apresentaram alterações ao EEG.

Hematoma - De 40 pacientes com hematoma intracraniano, foram realizados exames pré-operatórios em 31 (32 exames) e pós-operatórios em 35 pacientes (55 exames). Dos 31 pacientes com exames pré-operatórios, apenas dois tiveram EEGs normais. O quadro 8 assinala a distribuição dos EEGs pré-operatórios e pós-operatórios, segundo a anormalidade eletrencefalográfica e o tipo de hematoma. Dominam as ALCD e ALCF, porém há destacada associação com as depressões focais $(19,35 \%)$. Estas ocorreram em apenas $5,18 \%$ dos traumatizados sem hematoma, o que é significativamente diferente do grupo com hematoma $\left(\chi^{2}=9,606 ; 1\right.$ G.L.; $0,01>P>$ 
$>0,001)$. E ainda, quando comparamos os pacientes com hematoma aos sem essa intercorrência há diferença estatisticamente significante ( $\chi^{2}$ com correção de Yates $=13,687 ; 1$ G.L.; $P<0,001$, mostrando a maior freqüência de anormalidades no grupo com hematoma (Quadro 9).

Em 20 pacientes, o EEG permitiu lateralização adequada $(66,66 \%)$, sendo que em um deles não havia outros dados ao exame neurológico que a sugerissem. A lateralização foi impossivel em 5 pacientes e errada em 3 , não havendo nestes, ao exame neurológico, dados de lateralização. No sentido de procurar distinguir o sofrimento focal de 4 pacientes associando ou não à existência de possivel hematoma, foi feita injeção endovenosa de $10 \mathrm{mg}$ de diazepam. Esses pacientes mostraram atenuação das anormalidades lentas contínuas focais, sugerindo inexistência de hematoma; o estudo angiográfico, realmente, não mostrou coleção sangüínea intracraniana.

Nos exames pós-operatórios houve maior proporção de EEGs normais, embora persistissem as anormalidades lentas continuas focais em porcentagem elevada $(55,55 \%)$.

\section{COMENTARIOS}

Dos 387 pacientes, $82(21,18 \%)$ apresentaram perda de consciência por mais de 24 horas, 40 tinham hematoma (10,33\%) e 96 mostraram sinais focais ao exame neurológico $(24,80 \%)$. Esses dados revelam a grande proporção de traumatismos crânio-encefálicos graves de nossa casuística, traduzindo certa seleção casual dos pacientes estudados.

Houve absoluto predomínio de pacientes do sexo masculino acima dos 10 anos de idade, explicado pela maior exposição aos acidentes do trabalho e conseqüentemente, aos acidentes de trânsito.

Quanto à idade, a menor proporção das anormalidades nas crianças com menos de 5 anos, ao contrário da experiência de Kellaway (1949) 11 e Strauss (1961) ${ }^{24}$, pode ser explicada pela menor gravidade do trauma de nossos pacientes nessa faixa etária. Já Kramarz (1962) ${ }^{13}$ encontra 247 EEGs normais em 300 pacientes com menos de 15 aros e 70 em 78 crianças com menos de 3 anos, porém sua casuistica não assinala pacientes com EEGs realizados no primeiro mês após o trauma. Além disso, a grande variabilidade individual torna dificil a caracterização patológica ou normal do EEG numa criança.

Por vezes um EEG é considerado como normal para a idade, porém a repetição do exame mostra melhora importante na organização ou na freqüência do ritmo de base ${ }^{23}$. Por isso, tem sido realçada a necessidade de estudos eletrencefalográficos longitudinais ${ }^{11}$ cesde os primeiros dias após o trauma ${ }^{21}$. Estas serviriam não só para avaliar a lesão na fase aguda, como também para ulteriormente servir de guia na correlação com determinados sintomas, mesmo que já tenha havido normalização do EEG.

A alta proporção das alterações focais parietoccipitais nas crianças com menos de 10 anos por nós assinalada, foi referida por vários autores, sendo interpretada como reação do cérebro imaturo ao trauma ${ }^{11,23}$ ou mesmo outras lesões ${ }^{14}, 17$, sendo, por vezes, também registradas em traumas leves sem grande valor prognóstico ${ }^{14,23}$. 
Tempo entre o EEG e o traumatismo - Uma idéia evolutiva das alterações eletrencefalográficas pode ser obtida ao analisarmos as percentagens de pacientes com primeiro exame anormal, nos diferentes periodos após o trauma. De $76,92 \%$ no primeiro dia do trauma, passou a 30,00\% entre 6 e 12 meses (Quadro 5). No entanto, deve haver uma seleção inevitável, desde que os pacientes assintomáticos não mais procuram assistência médica e, evidentemente, não são submetidos a novos exames. Inversamente, os pacientes que sofreram traumas mais severos, persistindo com sintomas e sendo acompanhados, contribuem para a. grande porcentagem de EEGs alterados após o primeiro ano. Esses dados são comparáveis aos de Williams (1941) ${ }^{27}$, que nas primeiras 4 semanas registrou 60 a $70 \%$ de anormalidades, menos de $30 \%$ no $7 .^{\circ}$ mês e $47 \%$ após os 2 anos. Silverman (1962) ${ }^{23}$, estudando pacientes com menos de 16 anos, encontrou 66\% de EEGs anormais nos exames realizados nas primeiras 4 semanas após o trauma $(76 \%$ na primeira semana e $55 \%$ nas semanas seguintes).

Levando em conta todos os EEGs e excluindo as repetições num mesmo periodo (Quadro 4), torna-se evidente a queda progressiva de EEGs anormais, sendo menor a proporção entre os 6 e 12 meses. A evolução dos vários tipos de anormalidade só poderia ser realmente conhecida pela repetição dos EEGs nos mesmos pacientes a intervalos regulares de tempo, condição de difícil obtenção.

Ao analisarmos o grupo geral de EEGs em relação ao tempo do trauma, há a possibilidade de que os mais tardios já correspondessem a pacientes com traumas mais severos. No entanto, observou-se queda acentuada da proporção de ALCD e ALCF entre o $1 .^{\circ}$ e o $6 .^{\circ}$ mês. $O$ mesmo aconteceu nos 28 pacientes seguidos eletrograficamente desde o EEG inicial até a normalização $(60,71 \%)$.

A crescente proporção das anormalidades paroxísticas, principalmente após os 6 meses, sugere que muitas anormalidades lentas contínuas evoluissem nesse sentido. Esses dados são similares aos de Robin (1963) ${ }^{21}$, que encontrou $50 \%$ de anormalidades aos 6 meses, ocasião em que a ALCD e ALCF já eram pouco freqüentes.

EEG e a gravidade do trauma - Vários autores 11, 13, 15, 23, 26, 27 mostraram a correlação entre a porcentagem de anormalidades ao EEG e a gravidade do trauma, caracterizada por seus componentes clínicos (duração da perda da consciência, sinais focais ao exame neurológico, líquido cefalorraqueano hemorrágico e trauma crânio-encefálico aberto).

Nos nossos 3 grupos quanto à perda de consciência (menos de uma hora, entre uma hora e 24 horas, e mais de 24 horas) houve nitida correlação entre EEG anormal e o tempo de perda de consciência $(47,45 \%, 66,66 \%$ e $86,58 \%$, respectivamente). Nos pacientes com perda de consciência por mais de uma hora foi encontrada anormalidade ao EEG em 77,93\%, enquanto Strauss (1961) ${ }^{21}$ assinalou-a em 33\%. Esta diferença pode ser explicada porque esse autor excluiu de sua casuística os pacientes com "problemas neurológicos grosseiros". 
A semelhança dos grupos sem perda de consciência e com perda de consciência por menos de uma hora pode ser em parte explicada pelo fato de a informação depender do paciente ou acompanhante, podendo em muitos casos ser questionada sua real existência. Nos demais grupos, muitos pacientes eram observados em hospital, tornando os dados mais seguros.

Quanto aos EEGs de pacientes em estado comatoso, é interessante a presença, em 4 deles, de elementos similares aos "spindles" do sono e ondas agudas do vertex, fato assinalado pela primeira vez por Chatrian et al. (1963) ${ }^{1}$ em 9 dentre 46 pacientes com distúrbio de consciência e que refletiria um estado funcional transitório decorrente de alterações da substância reticular mesencefálica. Em um caso, o EEG praticamente não podia ser distinguido de um traçado normal de vigília, fato também referido por Chase, Moreti e Prensky (apud Plum \& Posner - 1973) ${ }^{19}$.

Os sinais neurológicos focais, constituem nítido fator determinante de EEGs anormais $(83,33 \%$ de nossos pacientes) e referido também por outros autores $23,24,26$. Entretanto, na vigência de sinais focais ao exame neurológico, nem sempre as alterações focais ao EEG foram correspondentes na localização e intensidade, assim como as anormalidades focais eletrencefalográficas muitas vezes não tinham manifestações neurológicas nítidas associadas.

Também o líquido cefalorraqueano hemorrágico e o traumatismo crânio-encefálico aberto, na experiência de vários autores ${ }^{23,24,26}$ são acompanhados de grande número de EEGs anormais, semelhantemente à nossa casuística.

Já a presença de fratura simples de crânio é associada, em algumas séries $13,23,24$, a aumento de anormalidades, porém, Williams (1941) 27 não encontra diferença, desde que haja comparação adequada com traumas cranianos de igual gravidade. A nossa porcentagem $(68,29 \%)$ está próxima aos casos em que a fratura não foi comprovada.

Aparecimento de convulsões - Paillas et al. (1962) 18 encontraram epilepsia pós-traumática em $37 \%$ dos traumas abertos de crânio e em 9,4\% dos traumas fechados. Jennett (1962) ${ }^{9}$ assinalou epilepsia iniciada na primeira semana em menos de $5 \%$ dos casos e após a primeira semana em cerca de $5 \%$, sendo esse risco maior nos hematomas.

Dos nossos 337 pacientes com trauma fechado as crises convulsivas ocorreram em 31 casos $(9,19 \%)$. Dentre os 10 casos de trauma aberto de crânio, 4 apresentaram crises convulsivas, e dentre os 40 pacientes com hematoma, 8 apresentaram essa intercorrência. As crises generalizadas foram as mais freqüentes em nossa casuística, ocorrendo em 16 (39,53\%) dos 43 pacientes, semelhantes a Paillas et al. (1962) ${ }^{18}$, que as encontrou em $42 \%$ de seus 216 pacientes.

O EEG foi anormal em $81,39 \%$ dos nossos pacientes com convulsões, resultado também comparável ao de Paillas et al. (1962) ${ }^{18}$, que encontrou anormalidades em $90 \%$ dos casos. 
Courjon (1956) ${ }^{6}$ descreve, em 10 pacientes, atividade critica localizada, sem manifestação clínica concomitante. Pudemos observá-la em apenas um de nossos pacientes.

A previsão de epilepsia pelo EEG é difícil de ser feita, mas segundo Paillas et al. (1962) ${ }^{18}$, a repetição de registros a intervalos regulares pode fornecer dados para o prognóstico, em desacordo com Marshall \& Walker (1961) ${ }^{16}$, que negam o valor do EEG como indicador significativo de prognóstico.

Hematomas - A alta proporção de anormalidades eletrencefalográficas nos pacientes com hematoma (em torno de 90\%) torna improvável sua existência face a um paciente com EEG normal, porém não afasta a possibilidade $2,3,4,10,12$.

Não há uniformidade na descrição das anormalidades pelos vários autores $4,5,10$, porém as anormalidades focais constituídas por ondas lentas são as mais freqüentes ${ }^{4}$, e a presença associada de redução local de amplitude pode sugerir a presença de hematoma $3,5,10$.

Esta redução de amplitude seria ocasionada, segundo Jung (apud Chris$\operatorname{tian}^{2}$ ), pela interposição do hematoma entre o cérebro e os eletrodos, porém Chusid \& Gutierrez-Mahoney (1956) ${ }^{3}$ observaram pouca diferença entre os EEGs pré-operatórios e pós-operatórios precoces, sugerindo como causa as lesões de estruturas corticais e subcorticais por compressão, sofrimento vascular e edema secundários ou não ao hematoma ${ }^{10}$.

Em 30 pacientes com hematoma, conseguimos lateralização adequada em 20, errada em 3 e impossivel em 5 (Quadro 10). Apenas dois pacientes tiveram EEGs normais. A distribuição, segundo o tipo de hematoma e a possibilidade de lateralização, está assinalada no quadro 10 . Chusid \& Gutierrez-Mahoney (1964) ${ }^{4}$ encontraram lateralização correta em 19 de seus 29 pacientes, Courjon et al. $(1950)^{5}$ em 10 de seus 13 pacientes e Kaplan et al. (1956) ${ }^{10}$ em 26 dentre 37 pacientes. Infelizmente, esses autores não citam dados a respeito de lateralização errada.

\section{RESUMO E CONCLUSOES}

São analisados 551 eletrencefalogramas de 387 pacientes com traumatismo crânio-encefálico. Os achados eletrencefalográficos são correlacionados aos seguintes dados clinicos: idade, tempo após o trauma, duração da perda de consciência, presença de sinais focais ao exame neurológico, existência de traumatismo crânio-encefálico aberto ou hematoma, liquido cefalorraqueano hemorrágico ou fratura de crânio e aparecimento de convulsões.

O autor chega às seguintes conclusões: 1 - há nítida correlação entre as anormalidades eletrencefalográficas e o grau de lesão cerebral, caracterizado pelo tempo de perda de consciência, presença de sinais focais ao exame neurológico, presença de liquido cefalorraqueano hemorrágico e epilepsia pós-traumática; 2 - as anormalidades lentas contínuas focais parie- 
toccipitais são mais freqüentes na faixa etária com menos de 10 anos; 3 - há uma queda de proporção de eletrencefalogramas anormais particularmente entre o $6 .^{\circ}$ e o $12 .^{\circ}$ mês após o trauma; 4 - as anormalidades lentas contínuas difusas ou focais têm sua proporção diminuida particularmente após o primeiro mês depois do trauma; 5 - as anormalidades paroxísticas aumentam, em proporção, de modo nítido, após o $6 .^{\circ}$ mês depois do trauma; 6 - os pacientes com hematoma intracraniano apresentam grande proporção de anormalidades, particularmente as depressões.

Todos esses dados mostram a importância do eletrencefalograma na complementação do estudo clínico dos pacientes com traumatismo crânio-encefálico, principalmente quando é feita seqüência eletrencefalográfica, desde os primeiros dias após o trauma.

\section{SUMMARY}

The value of electroencephalogram in head injuries

The 551 electroencephalograms of 387 patients with head injury are analysed. The electroencephalographic findings are correlated to the following clinical data: age, time after the trauma, duration of unconsciousness, presence of localizing neurological signs, existence of open wounds or hematoma, bloody cerebrospinal fluid and post-traumatic epilepsy.

The autor came to the following conclusions: 1 - There is a clear correlation between the electroencephalographic abnormalities and the degree of cerebral injury characterized by the duration of unconsciousness, presence of localizing neurological signs, presence of bloody cerebrospinal fluid and post-traumatic epilepsy; 2 - slow wave activity over the parieto-occipital area is more frequent in the age group up to 10 years; 3 - the proportion of abnormal electroencephalograms decreases particularly after the 1st month post-trauma; 4 - diffuse or focal slow activity decreased principally after the 1st month post-trauma; 5 - paroxysmal abnormalities increase proportionaly after the 6 th month post-trauma; 6 - patients with intracranial hematoma present great proportion of abnormalities, particularly depressions.

This study demonstrates the importance of electroencephalographic tracings as a complementation to the clinical evaluation of head injury cases, chiefly early, and late follow-up tracings.

\section{REFERENCIAS}

1. CHATRIAN, G. E.; WHITE Jr., L. E. \& DALY, D. - Electroencephalographic patterns resembling those of sleep in certain comatose states after injuries to the head. Electroenceph. clin. Neurophysiol. 15:272, 1963.

2. CHRISTIAN, W. - Atlas de Electroencefalografia Clínica. Versão castelhana. Editorial Paz Montalvo, Madrid, 1971.

3. CHUSID, J. G. \& GUTIERREZ-MAHONEY, C. G. - Head injury and subdural hematoma: electroencephalographic studies. Electroenceph. clin. Neurophysiol. 1:496, 1949. 
4. CHUSID, J. G. \& GUTIERREZ-MAHONEY, C. G. - The electroencephalogram in head injuries with sub-dural hematoma. Neurology (Minneapolis) 6:11, 1956.

5. COURJON, J.; BONNET, H. \& ALLEGRE, G. - Etude electroencephalographique de 17 hématomes sous-duraux. Rev. Neurol. (Paris) 83:609, 1950.

6. GOURJON, J. - Les activités électriques critiques localisées dans les traumatismes craniens fermés récents. Valeur diagnostique et prognostique. Rev. Neurol. (Paris) 95:537, 1956.

7. DELAY, J. \& VERDEAUX, G. - Electroencéphalographie Clinique. Masson et Cie., Paris, 1936.

8. GIBBS, F. A. \& GIBBS, E. L. - Atlas of Electroencephalography, Vol. 3, Addison-Wesley, Massachusetts, 1964.

9. JENNETT, W. B. - Epilepsy After Blunt Head Injuries. William Heinemann, London, 1962.

10. KAPLAN, H. A.; HUBER, W. \& BROWDER, J. - Electroencephalogram in subdural hematoma: a consideration of its pathophysiology. J. Neuropat. Exp. Neurol. 14:66, $195 \overline{6}$.

11. KELLAWAY, P. - Head injury in children. Electroenceph. clin. Neurophysiol. 1:497, 1949.

12. KILOH, L. G. \& OSSELTON, J. W. - Clinical Electroencephalography. Butterworths, London, 1966.

13. KRAMARZ, P. - L'électroencéphalogramme systématique dans les traumatismes cranio-encéphaliques de l'enfant. Ann. Med. Lég. 42:216, 1962.

14. LAGET, P. \& SALBREUX, R. - Atlas D'Electroencéphalographie Infantile, Masson et Cie., Paris, 1967.

15. LERIQUE-KOECHLIN, A. \& LOSSKY-NEKHOROCHEFF, I. - Conclusions sur l'EEG de l'enfant après traumatisme crânien. Ann. Méd. Lég. 42:229, 1962.

16. MARSHALL, C. \& WALKER, A. E. - The value of electroencephalography in the prognostication and prognosis of post-traumatic epilepsy. Epilepsia 2:138, 1961.

17. MEYER-MICKLEIT, R. W. \& SCHNEIDER, E. R. - The EEG in residual epilepsy after brain lesions at birth and in childhood. Electroenceph. clin. Neurophysiol. 13:658, 1961.

18. PAIllas, J. E.; COURSON, B.; NAQUET, R. \& PAILlaS, N. - Epilepsie posttraumatique: considérations sur une série de 216 observations. Sem. Hop. Paris. 38:1191, 1962.

19. Plum, F. \& POSNER, J. B. - Lo Essencial del Estupor y Coma. Versão castelhana. El Manual Moderno, México, 1973.

20. PUPO, P. P. \& PIMENTA, A. M. - A electrencefalografía como meio semiológico nos traumatismos crânio-encefálicos. Neurobiol. (Recife) 10:13, 1947.

21. ROBIN, A. - Quelques particularitées de l'EEG dans les traumatismes crâniens. Acta Neurol. Psychiat. Beig. 63:1034, 1963.

22. SELIGMANN, F. - Sequelles neurologiques et electroencphalographiques de $\mathbf{5 2}$ traumatismes crâniens chez l'enfant et indications therapeutiques. Rev. Neuropsychiatrie Infant. 13:439, 1967.

23. SILVERMAN, D. - Electroencephalographic study of acute head injury in children. Neurology (Minneapolis) 12:273, 1962.

24. STRAUSS, H. - Diagnostic value of the electroencephalogram in concussion and its sequelae. New York state J. Med. 61:1884, 1961.

25. ULETT, G. A. - Clinical and experimental studies of mild head injuries. Electroenceph. clin. Neurophysiol, 1:496, 1949.

26. WILLIAMS, D. - The electroencephalogram in acute head injuries. J. Neurol. Neurosurg. Psychiat, 4:107, 1941.

27. WILLIAMS, D. - The electroencephalogram in chronic post-traumatic states. J. Neurol. Neurosurg. Psychiat. 4:131, 1941. 